\title{
A generalized equation approach for hyoid bone displacement and penetration-aspiration scale analysis
}

\author{
Zhenwei Zhang $^{1} \cdot$ Atsuko Kurosu $^{2} \cdot$ James L. Coyle $^{2} \cdot$ Subashan Perera $^{3} \cdot$ Ervin Sejdić $^{1}$
}

Received: 30 October 2020 / Accepted: 4 May 2021

Published online: 07 June 2021

(c) The Author(s) 2021

OPEN

\begin{abstract}
Swallowing physiology includes numerous biomechanical events including displacement of the hyoid bone, which is a crucial component of airway protection and opening of the proximal esophagus. The objective of this study was to evaluate the potential relations between the trajectory of hyoid bone movement and the risk of airway penetration and aspiration during a videofluoroscopic swallowing study. Two hundred sixty-five patients were involved in this study, producing a total of 1433 swallows of various volumes consisting of thin liquid, nectar-thick liquid, and solids during a fluoroscopic exam. The anterior and posterior landmarks of the body of the hyoid bone were manually marked in each frame of each fluoroscopic video. Generalized estimation equations were applied to evaluate the relationship between penetration-aspiration scores and mathematical features extracted from the hyoid bone trajectories, while also considering the influence of other independent variables such as age, bolus volume, and viscosity. Our results indicated that penetration-aspiration scores showed a significant relation to age. The maximum anterior (horizontal) displacement of the anterior hyoid bone landmark was significantly associated with the penetration-aspiration scores. Differences in the displacement of the hyoid bone are useful observations in airway protection.
\end{abstract}

\section{Article highlights}

(1) In this work, the potential relations between the trajectory of hyoid bone movement and the risk of airway penetration and aspiration during a videofluoroscopic swallowing study were evaluated.
(2) We extracted features from the hyoid bone trajectories and applied generalized estimation equations to investigate their relationship to penetration-aspiration scales.

(3) The results showed that the maximum anterior (horizontal) displacement of the anterior hyoid bone landmark was significantly associated with the penetration-aspiration scales.

Keywords Dysphagia $\cdot$ Hyoid displacement $\cdot$ Generalized estimating equation Swallowing

\footnotetext{
Manuscript submitted Dec 6, 2020. Research reported in this publication was supported by two grants from the Eunice Kennedy Shriver National Institute of Child Health \& Human Development of the National Institutes of Health under Award Number R01HD092239, while the data was collected under Award Number R01 HD074819. The content is solely the responsibility of the authors and does not necessarily represent the official views of the National Institutes of Health.
}

$\triangle$ Ervin Sejdić, esejdic@pitt.edu|'Department of Electrical and Computer Engineering, Swanson School of Engineering, University of Pittsburgh, Pittsburgh, PA 15261, USA. ${ }^{2}$ Department of the Communication Science and Disorders, School of Health and Rehabilitation Sciences, University of Pittsburgh, Pittsburgh, PA 15260, USA. ${ }^{3}$ Division of Geriatric Medicine, Department of Medicine, University of Pittsburgh, Pittsburgh, PA 15261, USA. 


\section{Introduction}

Swallowing is a complex neuromuscular process needed for proper daily nutrition. There are four main stages of the process of swallowing: the oral preparatory stage, oral transit stage, pharyngeal stage and esophageal stage. Dysphagia is a term used to describe any swallowing dysfunction [1-4] that causes subjective discomfort or objective impairment in the formation or transportation of a bolus safely and completely from mouth to the stomach without entering the airway [5] in one or more of these four stages. In the healthy swallow, the upper aerodigestive tract alternates between respiratory (breathing) and digestive (swallowing) functions within a very short duration of less than 2 s. Airway compromise in dysphagic people is caused by the mistiming of these airway protective events enabling swallowed material to be misdirected into the upper laryngeal or lower tracheal airway (penetration, aspiration respectively). As a consequence of aspiration, patients can develop adverse medical complications such as airway obstruction (choking) and aspiration pneumonia [6-9], a leading cause of dysphagia-related mortality. These serious consequences, caused by dysphagia, impact both the health and the quality of patients' daily life. Annually in the United States, approximately $4 \%$ of adults have swallowing-related disorders [10]. It is estimated that $12-13 \%$ of patients in short-term acute care hospitals and around $60 \%$ of nursing home occupants have swallowing difficulties [6]. Rapid identification of dysphagia along with timely diagnosis and management of the swallowing disorder is necessary to mitigate the risk of these preventable adverse outcomes. Due to the intricate neuromuscular coordination of the swallowing process, swallowing disorders commonly appear in patients with neurological diseases such as stroke, Parkinson's disease [11], head/neck cancer and brain injuries [12], and many other conditions. Many symptoms associated with dysphagia are poorly recognized by clinicians and other observers, such as family members, who are not trained to recognize them. Specialized dysphagia clinicians are often not consulted until a preventable dysphagia-related adverse event has already occurred. Delayed detection of clinically significant dysphagia is particularly problematic in under served settings and clinics that are not staffed with specialists.

There are two principal diagnostic examinations widely accepted as gold standard imaging tests used in the diagnosis of dysphagia and treatment planning: videofluoroscopic swallowing studies (VFSS), commonly called modified barium swallow studies, and fiberoptic endoscopic evaluation of swallowing (FEES). The VFSS was first described in the early 1980s by Logemann [13] though developmental iterations of the examination were described in the literature as long ago as the late 1970s. FEES, which is an adaptation of typical fiberoptic nasopharyngoscopy, was first described in 1988 by Langmore [14] as the miniaturization and refinement of endoscopic instrumentation grew. Although both methods require specialized training to ensure safe and appropriate testing, the VFSS examination is in more widespread use given that its adoption precedes that of FEES, and because the only specialized equipment necessary for the VFSS (i.e., a fluoroscopy unit) is present in any healthcare institution with a radiology department. Each examination procedure provides information regarding swallowing anatomy and physiology that are complementary to one another though each is regarded as a current gold standard. This issue is debatable because each examination, though providing ample information with which to form diagnostic impressions, has advantages and limitations based on the technology used. For example, VFSS is considered more comprehensive because it provides continuous viewing of the entire swallowing mechanism during swallow, but is limited by the need of limit x-ray exposure to the patient, while FEES provide excellent high-resolution images but of a very narrow field of view to which the examiner is blinded due to the momentary collapse of the pharynx during the important pharyngeal stage of swallowing. VFSS, unlike FEES, provides continuous imaging data of all upper aerodigestive structures that are active during swallowing in the oral, pharyngeal, laryngeal and upper esophageal regions, while FEES imaging examines an approximately $2-4 \mathrm{~cm}$ view of the larynx and pharynx before the onset and after the conclusion of the pharyngeal swallow. Both tests provide excellent information regarding the presence, severity and immediate results of aspiration in patients with dysphagia (e.g., whether the patient produced an airway protective reflex such as a cough), though only VFSS is capable of evaluating the underlying contributions of impaired lingual, pharyngeal, hyolaryngeal, and upper esophageal sphincter function underlying airway protection $[15,16]$. The majority of data produced over the past $40+$ years, delineating the normal biomechanical swallowing sequence of events which are largely undetectable with FEES, has been generated using VFSS imaging. During VFSS, a patient is seated before an X-ray machine and instructed to swallow different liquids and/or foods mixed with barium [17]. Typically, the swallowing assessment is carried out by a radiologist and a speech-language pathologist [18]. Examiners observe the biomechanical displacement of numerous oropharyngeal structures such as the hyoid bone, pharyngeal walls, larynx and epiglottis, tongue, 
and upper esophageal sphincter to determine the nature of impaired swallowing and identify interventions best suited to alleviate them. During these assessments, the timing, patterns and distance of displacement of these structures in producing a flow of the radio-opaque boluses through the upper aerodigestive tract are examined and discrete measurements can be observed by clinicians. These clinicians can then evaluate swallowing integrity and identify the effects of various interventions that lead to aspiration and its consequences. The evaluations include compensatory alteration of patient posture, and modification of bolus textures or bolus volumes on swallowing physiology $[19,20]$.

The kinematics of swallowing physiology have been well described in the literature and the components germane to the current investigation will be briefly summarized here [22]. Each bolus is propelled through the oral cavity to the oropharynx where a fairly predictable sequence of pharyngeal collapse, hyolaryngeal excursion, airway closure and UES opening are observed. The hyolaryngeal excursion is a key component of swallowing physiology because it delivers traction forces to the hyolaryngeal complex that leads to closure of the laryngeal vestibule and distention of the UES, provided that all other components of the pharyngeal stage are intact and occur in the correct temporal order. Superior (vertical) and anterior (horizontal) displacement of the hyolaryngeal complex (HLC), which occurs typically following a reduction in the upper esophageal sphincter (UES) resting tone through vagal inhibition, is responsible for delivering traction forces to the UES to enable UES opening for clearance of swallowed material, as well as for displacement and reconfiguration of the upper airway to prevent aspiration. Six pairs of primary suprahyoid muscles originating on the mandible, skull base and tongue, and several pairs of long pharyngeal muscles (e.g., palatopharyngeus) contribute to the net displacement of the HLC while a single paired muscle (i.e. thyrohyoid) contributes to the shortening of the larynx during swallowing. When contraction of all suprahyoid muscles is summated, a net superior and anterior vector of $\mathrm{HLC}$ traction forces is delivered to the anterior wall of the UES, which is shared as the posterior wall of the larynx. The combined net action of these muscles is the superior (vertical) and anterior (horizontal) displacement of the HLC during swallowing [23]. The hyoid bone, a radiographically prominent landmark of the HLC, has been shown in numerous kinematic analyses to move both vertically and horizontally, in patterns that vary slightly from person to person, and then return to the starting position when muscular contractions subside after single swallows [24]. This displacement pattern reconfigures the upper aerodigestive tract to facilitate closure of the laryngeal vestibule and in the presence of neurally modulated relaxation of the UES, applies traction to the anterior wall of the UES facilitating, opening the esophagus for food to be delivered into the esophagus and subsequently the stomach. Evaluation of anterior hyoid excursion from VFSS images is considered important in evaluating the nature of the swallowing impairment and the extent to which impaired excursion of the HLC contributes to airway compromise, inefficient clearance into the esophagus, and post-swallow pharyngeal residue. Inadequate anterior hyolaryngeal displacement leads to incomplete laryngeal vestibule closure and inadequate traction forces on the UES, which, when combined, lead to airway penetration and incomplete opening of the UES. The separation of portions of a bolus is due to premature UES closure which can subsequently be aspirated after the swallow [18]. Previous studies that investigated the associations between hyoid movement and the presence of penetration/aspiration produced mixed results. Steele et al. reported a positive correlation between reduced anterior hyoid movement and the presence of aspiration and penetration [25]. Feng et al. reported that the maximal hyoid bone displacement during swallowing was reduced among older patients with aspiration when compared to those who did not aspirate $(P<0.05)$ [26]. On the other hand, Kim et al. indicated that there was no difference in anterior hyoid displacement between aspirators and non aspirators in patients with stroke $(P=0.43)$ [27]. Seo et al. investigated the maximal displacement and velocity of hyoid bone and larynx and indicated that there was no relationship between maximum hyoid displacement and the presence of penetration/aspiration in 68 patients with stroke $(P>0.1)$ [28]. Molfenter et al. studied hyoid bone movement duration and position during the swallow. They reported there was no difference on hyoid displacements between aspirators and non-aspirators from 178 swallows obtained from 42 stroke patients $(P>0.05)$ [29].

Therefore, we sought to investigate the motion of the hyoid bone by analyzing both kinematic displacements and mathematical trajectory features of those displacements during swallowing in 265 patients with dysphagia to determine whether there are relationships between characteristics of hyoid bone trajectory and a score on the penetration aspiration scale (PAS) [30]. We hypothesized that the hyoid's trajectory features would differentiate between normal PA scale scores indicating no airway penetration or transient shallow laryngeal penetration (score of 1-2) and abnormal PA scale scores indicating shallow laryngeal penetration with post swallow laryngeal residue through deep laryngeal penetration and all aspiration events (scores of 3-8). A generalized estimate equation model was built to test our hypothesis based on trajectories extracted from VFSS images during various swallowing tasks. If the findings of this methodological 
study are confirmed, the analysis of hyoid trajectory features would be a useful additional component to characterize the nature of penetration and aspiration in some patients with dysphagia, and to inform clinicians regarding the appropriate interventions to restore a more normal $\mathrm{HLC}$ displacement during swallowing.

The paper is organized as follows: Sect. 2 describes data collection, image analysis methods, feature extraction methods, and statistical analysis methods investigated in this study. The analysis and results from the generalized equation are given in Sect. 3. We discuss our findings between features from the hyoid bone movement and penetration-aspiration in Sect. 4. Finally, we present the conclusions of this study.

\section{Methods}

\subsection{Data acquisition}

Two hundred and sixty five patients with clinical suspicion of dysphagia underwent videofluoroscopic examination at the Presbyterian University Hospital of the University of Pittsburgh Medical Center (Pittsburgh, Pennsylvania). The protocol for this study was approved by the Institutional Review Board at the University of Pittsburgh and all participants provided informed consent. The average age of the subjects was $64.83 \pm 13.56$ years old, and the age range was from 19 to 94 . Patients with tracheostomy or anatomic disruption or abnormalities of the head and neck were excluded. Patients swallowed boluses of liquids of different consistencies and volumes as well as cookies during VFSS. To mitigate the potential that the research data collection protocol would interfere with clinical management, the number and order of the swallow trials for each consistency and volume were determined by the examining clinician based on the patient's history and clinical evaluation observations. The following consistencies were used: E-Z-EM Canada, Inc. Varibar thin (Bracco Diagnostics, Inc.) ( $<5$ cPs viscosity), Varibar nectar (300 cPs viscosity), Varibar pudding (5000 cPs viscosity), and Keebler Sandies Mini Simply Shortbread Cookies (Kellogg Sales Company). 1136 swallows were recorded in the lateral/sagittal plane with an additional 252 performed in a head-neck flexion position (chin down). Patients swallowed boluses administered by a spoon in 3-5 mL volumes, or self-administered boluses from a cup at a self-selected, comfortable volume.

Fluoroscopy was set at 30 pulses per second (full motion) and video images were acquired at 60 frames per second by a video card (AccuStream Express HD, Foresight Imaging, Chelmsford, MA) and recorded to a hard drive with a LabVIEW program. The videos were made into two-dimensional digital movie clips of $720 \times 1080$ resolution, and down-sampled it to $30 \mathrm{frames} / \mathrm{s}$ to eliminate duplicated frames.

\subsection{Image analysis}

Each video sequence contained one swallow which was defined as the duration between the frame at which the head of the bolus reached the lower mandibular margin to when the tailing end (tail) of the bolus passed the upper esophageal sphincter (UES). The anterior and posterior landmarks of the body of the hyoid bone were plotted in each video frame using MATLAB (R2015b, The MathWorks, Inc., Natick, MA, USA), as shown in Fig. 1. A coordinate system was created for each patient based on anatomical landmarks of the vertebral column to measure the vertical and horizontal displacements of the hyoid landmarks. The anterior-inferior corner of the fourth cervical vertebral body was plotted as the origin, and then the $y$ axis

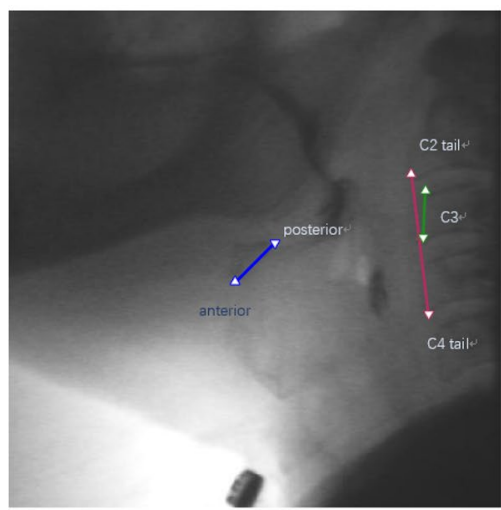

(a) Markers for anterior hyoid bone, posterior hyoid bone, $\mathrm{C} 2$ landmark, C4 landmark and C3

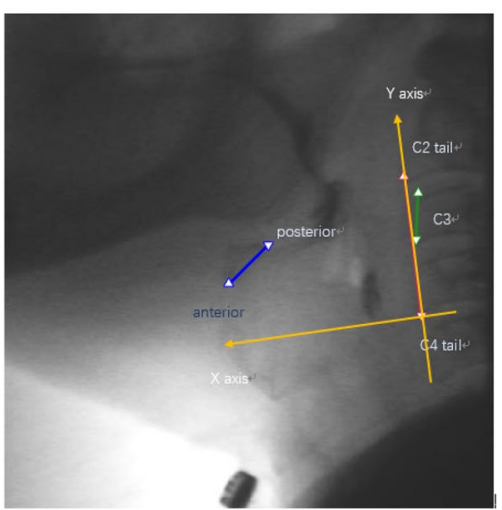

(b) Coordinate established based on the C2-C4

Fig. 1 The figures illustrate the markers for hyoid bone, C2, C3, C4 and how to establish the coordinate for hyoid bone trajectory 
was plotted as a straight line connecting this origin to the anterior-inferior corner of the second cervical vertebra. The $x$-axis is the straight line perpendicular to the $y$-axis and intersecting with the origin $[27,29]$. All distance numbers were first measured as the actual distance in image pixels. Then, to normalize participants of different heights to a common anatomic referent, the distance between the anterior-inferior and the anterior-superior corners of the third cervical vertebral body was set as a reference scale, referred to as "one C 3 unit".

Blinded to the hyoid trajectory results, two trained clinicians scored the presence/degree of penetration/aspiration from the 1433 swallows using the 8-point penetration aspiration scale [30] with a priori inter- and intra-rater reliability with ICCs of 0.99. Among these swallows, 1129 swallows had PAS of 1 or 2 (normal airway protection) and 304 swallows had PAS greater or equal to 3 (abnormal airway protection). The mean and standard deviation of PAS of all 1433 swallows are $2.117 \pm 1.580$.

Three raters trained in swallow kinematic analysis identified the anterior and posterior part of the hyoid bone in each frame during video analyses. To establish the interand intra-rater reliability of the hyoid bone annotations, $10 \%$ of the videos were re-analyzed with ICCs over 0.99 .

\subsection{Feature extraction}

We constructed six discrete series to represent hyoid bone motion trajectory: the motion along the $\mathrm{x}$ and $\mathrm{y}$ axis of the anterior-inferior and posterior-superior margin landmark of the body of the hyoid bone, the changes along the $y$-axis of the anterior and posterior margin of the hyoid bone, and the distance series of anterior superior/and posterior margin of the hyoid bone. The distance series was constructed by the Euclidian distance between the starting point and every subsequently plotted point. This series shows how consecutive points move closer or farther from the reference point. The distance series can be written as:

$D_{i}=\sqrt{\sum_{j=1}^{2}\left(X_{i j}-X_{0 j}\right)^{2}}$

In our investigations, independent variables of each VFSS examination such as patient age, bolus viscosity and size based on whether a spoon or cup was used to administer the bolus, were used. Furthermore, to capture the key statistical differences between series, the following mathematical features were extracted. Here, $n$ represents the length of the trajectory data series, and $x_{i}$ represents the ith data point in the series.
- Total length in pixels in the $x$ and $y$ plane minus $C 3$ which represents the $\mathrm{C} 3$ adjustment

- Median point in pixels of the series after C3 normalization

- Mean and standard deviation of hyoid bone movement for each plane

- Number of values below and above the mean for each plane

- Minimum and maximum hyoid bone position for each plane

- Minimum at first movement and maximum point of hyoid bone for entire hyoid movement

- The total duration of hyoid bone movement for each plane

- Sum over the absolute difference between subsequent time series values:

$$
\sum_{i=1}^{n-1}\left|x_{i+1}-x_{i}\right|
$$

- Mean of the absolute value of consecutive changes in the series $x$ :

$$
\frac{1}{n} \sum_{i=1}^{n-1}\left|x_{i+1}-x_{i}\right|
$$

- Skewness quantifies how symmetrical the amplitude distribution is, which can be computed as follows:

$$
\frac{\frac{1}{n} \sum_{i=1}^{n}\left(x_{i}-\bar{x}\right)^{3}}{\left[\frac{1}{n-1} \sum_{i=1}^{n}\left(x_{i}-\bar{x}\right)^{2}\right]^{\frac{3}{2}}}
$$

- Kurtosis measures whether the distribution is peaked or flat relative to a normal distribution, which can be expressed as follows:

$$
\frac{\frac{1}{n} \sum_{i=1}^{n}\left(x_{i}-\bar{x}\right)^{4}}{\left[\frac{1}{n} \sum_{i=1}^{n}\left(x_{i}-\bar{x}\right)^{2}\right]^{2}}
$$

\subsection{Statistical analysis}

A generalized estimating equations (GEE) model is popularly applied for clustered data in clinical studies. It is an extension of the quasi-likelihood approach [31]. The method was employed to construct a function of the feature set to match the outcome. The data are assumed to be dependent within subjects and independent between subjects. This model is quite useful with longitudinal data, which accounts for correlations between repeated measures on the same participant [32]. A GEE model assumes 
a relationship between $E(Y)$ and $\operatorname{Var}(Y)$ rather than a specific probability distribution for $Y$. A GEE model provides the best guess for the variance-covariance structure $\left(Y_{1}, Y_{2}, \ldots, Y_{T}\right)$ by a linear predictor linking each marginal mean [33]. $Y_{i t}$ represents the category for each subject $i$, measured at different time points $t$. The working correlation matrix is applied to guess for the correlation structure among $Y_{t}$. An exchangeable correlation structure is applied here, which is a useful structure when correlations are small, which treats $\operatorname{Corr}\left(Y_{i} s, Y_{i} t\right)$ as identical for all pairs $s$ and $t$. The GEE model assumes a probability distribution for each marginal distribution and provides reasonable estimates and standard errors. The GEE model estimates are obtained by using an iterative algorithm as there are no closed-form solutions.

In our studies, PAS has a skewed non-normal distribution and our data consist of multiple swallows from each participant, making common statistical techniques such as (generalized) linear models and classification/regression trees not readily applicable. Therefore, we employed the GEE model with low (1-2) or high (3-8) PAS as the dichotomous dependent variable, a binomial distribution, a logit link function and an exchangeable working correlation structure to predict the probability of a high PAS. Age, swallow type, viscosity, volume/utensil, and head position were used in the model as independent variables based on face validity. In addition, we used an independent variable forward selection approach to identify a parsimonious set of trajectory variables using a criterion of $P=0.05$ entry into the model. Using the final model, we obtained odds ratios, and their $95 \%$ confidence intervals and statistical significance for each independent variable. Also, to assess the concordance between predicted and observed high PAS, we created subgroups of swallows based on the predicted probability deciles and examined the actually observed percentage of high PAS swallows within each decile. SAS ${ }^{\circledR}$ version 9.3 (SAS Institute, Inc., Cary, North Carolina) was used for all statistical analyses with GENMOD procedure for obtaining the main results.

\section{Results}

The generalized estimating equation is built to estimate the relation between various features and PAS. Table 1 displays patient and swallows' characteristics. The changes in unbalanced data for the viscosity and head position are explained by changes in head position and various liquid volumes and viscosities during the swallow examination, based on clinical need. Table 2 provides an overview of the contribution of important variables with entry criterion 0.05 , based on the model estimate, odds ratio, and $P$ value. The independent characteristics forced into the model, regardless of their $P$ value, are basic information data: age, swallow type, viscosity, utensil, sex, head position and swallow duration. Patients may have multiple swallows during the examination when some of the bolus remains in the oral cavity or pharynx after the first swallow. "Multiple(1)" indicates the first swallow and "multiple(2)" indicates the following swallows. Table 3 indicates significant features related to PA scores in univariate analysis. Table 2 reveals the features that were statistically significant at $P<0.05$, providing strong contributions to the model related to the PAS. Independent variables of the patient and swallow condition, such as older age, first multiple swallows, and thin liquid viscosity, were significantly associated with higher PS, and the hyoid horizontal displacement independent variable was also significantly associated with higher PAS. More details of hyoid bone maximum displacement can be found in the appendix.
Table 1 Clinical information of the patients and swallows

\begin{tabular}{|c|c|c|c|c|c|}
\hline \multicolumn{2}{|l|}{ Catagories } & \multirow{2}{*}{$\begin{array}{l}\text { Values } \\
64.83 \pm 13.56\end{array}$} & \multicolumn{2}{|l|}{ Catagories } & \multirow{2}{*}{$\frac{\text { Values }}{879}$} \\
\hline Age & & & Viscosity & Thin & \\
\hline Total swallows & & 1434 & & Nectar & 405 \\
\hline \multirow[t]{2}{*}{ Sex } & Male & 155 & & Pudding & 94 \\
\hline & Female & 110 & & Cookie & 42 \\
\hline \multirow[t]{3}{*}{ Utensil } & Spoon & 594 & & Not recorded & 13 \\
\hline & Cup & 832 & Type & Single & 498 \\
\hline & Not recorded & 7 & & multiple(1) & 360 \\
\hline \multirow[t]{3}{*}{ Head position } & Neutral & 1136 & & multiple(2) & 534 \\
\hline & Chin down & 252 & & Not recorded & 41 \\
\hline & Not recorded & 45 & & & \\
\hline
\end{tabular}

multiple(1) indicates the first swallow in the multiple swallow and multiple(2) indicates the subsequent swallows
SN Applied Sciences 
Table 2 Final model with forward selection with 0.05 entry criterion
Table 3 Univariate analysis of extracted hyoid bone features

\begin{tabular}{llllll}
\hline Parameter & Estimate & $P$ value & Odds ratio & $\begin{array}{l}\text { Odds ratio } \\
\text { lower }\end{array}$ & $\begin{array}{l}\text { Odds } \\
\text { ratio } \\
\text { higher }\end{array}$ \\
\hline Age & 0.0265 & 0.0178 & 1.03 & 1.00 & 1.05 \\
Type: single & -0.4435 & 0.0708 & 0.64 & 0.40 & 1.04 \\
Type: multiple(1) & 0.4545 & 0.0040 & 1.58 & 1.16 & 2.15 \\
Type: multiple(2) & 0.0000 & - & 1.00 & 1.00 & 1.00 \\
Sex: male & 0.1398 & 0.6998 & 1.15 & 0.57 & 2.34 \\
Sex: female & 0.0000 & - & 1.00 & 1.00 & 1.00 \\
Viscosity: thin & 1.2862 & 0.0096 & 3.62 & 1.37 & 9.58 \\
Viscosity: nectar & 0.7049 & 0.1664 & 2.02 & 0.75 & 5.49 \\
Viscosity: pudding & -0.5334 & 0.3789 & 0.59 & 0.18 & 1.92 \\
Viscosity: cookie & 0.0000 & - & 1.00 & 1.00 & 1.00 \\
Utensil: spoon & 0.1622 & 0.3538 & 1.18 & 0.83 & 1.66 \\
Utensil: cup & 0.0000 & - & 1.00 & 1.00 & 1.00 \\
Head position: neutral & 0.0994 & 0.7104 & 1.18 & 0.65 & 1.87 \\
Head position: chin down & 0.0000 & - & 1.00 & 1.00 & 1.00 \\
Swallow duration & -0.0004 & 0.9549 & 1.00 & 0.99 & 1.01 \\
Maximum displacement in & -0.0583 & 0.0064 & 0.94 & 0.90 & 0.98 \\
$\quad$ horizontal direction & & & & & \\
\hline & & & & & \\
\hline & & & & &
\end{tabular}

\begin{tabular}{lcccc}
\hline Variable features & Estimate & $P$ value & Lower CL & Upper CL \\
\hline Mean of the absolute value of consecutive changes in x2 & -1.479 & 0.0012 & -2.373 & -0.589 \\
Variance of x2 & -0.029 & 0.0024 & -0.047 & -0.010 \\
Standard deviation of x2 & -0.217 & 0.0012 & -0.348 & -0.086 \\
Maximum point of x2 & -0.056 & 0.0029 & -0.093 & -0.019 \\
Sum over the absolute difference in x2 & -0.030 & 0.0017 & -0.049 & -0.114 \\
Maximum point of x1 & -0.074 & 0.0005 & -0.115 & -0.032 \\
Variance of x1 & -0.041 & 0.0005 & -0.065 & -0.018 \\
Mean of the absolute value of consecutive changes in x1 & -1.527 & 0.0006 & -2.400 & -0.654 \\
Standard deviation of x1 & -0.275 & 0.0003 & -0.425 & -0.124 \\
Sum over the absolute difference in x1 & -0.032 & 0.0021 & -0.052 & -0.017 \\
Mean of x1 & -0.071 & 0.029 & -0.132 & -0.010 \\
Minimum to maximum displacement of x1 & -0.086 & $<0.0001$ & -0.127 & -0.045 \\
Maximum to minimum displacement of x2 & -0.046 & 0.0182 & -0.084 & -0.008 \\
x1: anterior-inferior displacement in horizontal direction & x2: posterior-superior displacement in \\
& horizontal direction & \\
\hline
\end{tabular}

The analysis was implemented on variables one a time. The table only listed the significant features results associated to higher PAS. The results indicated that only the features in horizontal displacement had the significant associations to PAS

\section{Discussion}

In the present study, we sought to investigate whether a relationship exists between hyoid bone displacement features and examination condition variables on airway protection as measured by the PAS. We evaluated not only the maximal distance and velocity of the hyoid bone, but also features extracted from the trajectory of the hyoid bone. We included information such as age, bolus volume, swallow type (single/multiple), and head position as the necessary variables in the GEE model, and used forward selection to choose the important variables for the model prediction. Our results demonstrated that the maximum displacement of the hyoid bone in the horizontal direction was significantly related to PAS while all other trajectory features are not significantly related to PAS. We will 
discuss the significant feature related to PAS and compare the findings of basic variables with other contributions.

We tested the trajectory features extracted from the motion of the hyoid bone against variables such as age, bolus volume, viscosity, and head position. In univariate analysis, thirteen features show significance associated to higher PA scores. These features are all related to horizontal direction while none of the features from hyoid bone displacement in the vertical direction are significant with the PA scores. Then we applied feed forward selection in GEE variable selection. From the GEE model, we found that the maximum displacement of anterior-inferior hyoid bone has a significant relation to the PAS: smaller displacement was associated with higher PAS. Other features extracted from the hyoid bone displacement did not show significant associations with the PAS. The finding that horizontal displacement of the hyoid bone is significant but not the vertical displacement particularly matches the result found by Kim et al. They found that there was a significant difference between younger and older subjects for anterior displacement but not vertical displacement.

Steele et al. suggested the C2-C4 vertebral distance should be used to normalize the hyoid displacement measurements to account for individual anatomical size differences [34]. However, our results using the $\mathrm{C} 3$ height to normalize displacements to subject size agree with those of Steele et al., in which reduced anterior hyoid displacement (normalized by the C2-C4 distance), were associated with higher (worse) PAS [25]. On the other hand, Molfenter et al. [29] and Seo et al. [28] reported no difference in hyoid displacements between aspirators and nonaspirators in patients with stroke using the $\mathrm{C} 2-\mathrm{C} 4$ distance to normalize units.

Although Kim and McCullough used the anatomically normalized C3 unit, they tracked a different hyoid landmark (the superior-anterior aspect of the body of the hyoid bone) and found no difference in the maximum anterior displacement of hyoid between aspirators and non-aspirators in patients with stroke [27]. This methodological difference may explain the discrepancy in our results and the study by Kim and McCullough. The inconsistent use of normalization measures may explain variability in hyoid displacement measurements [29], for which further investigations are needed to clarify this disagreement.

Compared to prior research, our study has different methodology. In prior research, they grouped subjects based on judgment of whether they are patients or healthy subjects $[28,35]$. Our study investigated the relationship between hyoid displacement and the PAS at the swallow level. Therefore, it is worthwhile to evaluate each swallow to account for variability in hyoid displacement within individuals, as well as to determine whether the deployed research methods can detect relationships between hyoid movement patterns and airway protection during swallowing. This is relevant given the frequency and severity of laryngeal penetration and/or aspiration are key diagnostic components for guiding dysphagia treatment. These methods can be deployed in future studies designed to characterize differences in hyoid displacement-PAS relationships in groups of patients with different diagnoses.

Age is a significant influence on PAS ( $P$ value $<0.05)$. We found that with each increased year in age, the risk of penetration increased $5 \%$. This finding is in general agreement with several previous studies [36]. Daggett et al. found that the percentage of penetration and aspiration dramatically increased with healthy subjects over 50 years old [37]. Steele et al. [34] reported that individuals over the age of 80 years old had more risk for penetration and aspiration. Robbins et al. concluded that age was associated with higher PAS [38].

Our results showed that pudding has the lowest risk for a higher PAS, followed by cookie, nectar and thin liquid, which is in concordance with several previous studies demonstrating that thicker boluses generally resulted in lower PAS, both in healthy people and patient groups [37, 39]. Logemann et al. in a study of 711 patients with dysphagia due to Parkinson's disease or dementia, similarly found that thin-liquid aspirators had nearly $50 \%$ reduction in aspiration with the thickest of liquids they administered [40]. Newman et al. [41] collected 33 articles related to the effect of bolus viscosity and indicated that increasing the viscosity from liquid to nectar and pudding can reduce the PAS and suggested that patients with oropharyngeal dysphagia can benefit from the increasing viscosity.

In this investigation, we considered as many as possible conditions for the multivariant model analysis, but we still have several points to improve. First, few studies introduced the deployment of chin down (head neck flexion) for participants and claimed that may affect swallowing. Future investigation should consider whether different chin down positions may influence penetration and aspiration. Next, we applied the spoon and cup in our data collection. The bolus size is approximately around $5 \mathrm{~mL}$ and $20 \mathrm{~mL}$, which is different from other investigations that carefully controlled the bolus size in their studies to increase internal validity. Our goal of the investigation is to study the association between hyoid bone movement features and PAS in clinical practice, as a measure of external validity. That is, we agree that there are plenty of studies indicating the effects of various independent variables on swallow physiology using strictly controlled, often unnatural (for each individual) conditions of swallowing that do not reflect the range of individual variability of swallow physiology. Thus the external and ecological validity of our methods are high in comparison to strictly controlled studies with high internal validity, and complement that 
evidence base. In addition, our result still matches the findings of other investigations, which can show the robustness of our findings. We used C3 to normalize linear distances to individual patient height, while different distance markers were applied in other studies. Rules based on different normalization methods should be investigated in future studies, and we are in the process of submitting our evidence equating the $\mathrm{C} 3$ linear distance to the $\mathrm{C} 2-4$ distance. Finally, several studies showed that obesity is one of the important factors in respiratory sleep disorders [42, 43] and hyoid bone movement may be affected by obesity and various diseases differently, which may lead to PAS variance. In this study, we focused on investigation of the relationship between mathematical hyoid bone displacement features (which are not a component of traditional kinematic analysis) and penetration-aspiration. Further investigations can be established based on obesity factors and various diseases' effects on penetration and aspiration during the swallow.

\section{Conclusion}

This study employed the generalized estimating equation model to investigate the association between the hyoid bone displacement and penetration and aspiration. We have shown that the maximum displacement of the anterior-inferior hyoid bone landmark is significantly related to PAS. Specifically, reduced maximum anterior displacement of the hyolaryngeal complex leads to a higher PAS. Furthermore, age has relation to PAS while the volume, viscosity, and head position show weak associations to penetration-aspiration. These findings suggest that analysis of the trajectory of the hyoid bone could provide useful diagnostic information for identifying patients with an elevated risk of penetration and aspiration. Further investigations based on the hyoid trajectory including other hyoid landmarks and hyoid rotational patterns should be performed to improve our understanding of the relationship between hyoid movement and risks of penetration and aspiration.

Funding This study was funded by two grants from the Eunice Kennedy Shriver National Institute of Child Health \& Human Development of the National Institutes of Health under Award Number R01HD092239, while the data was collected under Award Number R01HD074819. The content is solely the responsibility of the authors and does not necessarily represent the official views of the National Institutes of Health.
Declaration

Conflict of interest The authors have no conflict of interest to declare.

Ethical approval All procedures performed in studies involving human participants were in accordance with the ethical standards of the institutional and/or national research committee and with the 1964 Helsinki declaration and its later amendments or comparable ethical standards.

Informed consent Informed consent was obtained from all individual participants included in the study.

Open Access This article is licensed under a Creative Commons Attribution 4.0 International License, which permits use, sharing, adaptation, distribution and reproduction in any medium or format, as long as you give appropriate credit to the original author(s) and the source, provide a link to the Creative Commons licence, and indicate if changes were made. The images or other third party material in this article are included in the article's Creative Commons licence, unless indicated otherwise in a credit line to the material. If material is not included in the article's Creative Commons licence and your intended use is not permitted by statutory regulation or exceeds the permitted use, you will need to obtain permission directly from the copyright holder. To view a copy of this licence, visit http://creativecommons.org/licenses/by/4.0/.

\section{Appendix}

See Table 4

Table 4 Mean and standard deviation of the vertical and horizontal maximum displacement of both anterior and posterior part of the hyoid bone relative to the $\mathrm{C} 3$ marker

\begin{tabular}{lll}
\hline Maximum displacement & & Values \\
\hline Anterior & Horizontal & $0.769 \pm 0.27$ \\
& Vertical & $1.089 \pm 0.40$ \\
Posterior & Horizontal & $0.866 \pm 0.29$ \\
& Vertical & $1.052 \pm 0.37$ \\
\hline
\end{tabular}

\section{References}

1. Clavé P, Shaker R (2015) Dysphagia: current reality and scope of the problem. Nat Rev Gastroenterol Hepatol 12(5):259-270

2. Dudik JM, Coyle JL, El-Jaroudi A, Sun M, Sejdić E (2016) A matched dual-tree wavelet denoising for tri-axial swallowing vibrations. Biomed Signal Process Control 27:112-121

3. Miller AJ (2008) The neurobiology of swallowing and dysphagia. Dev Disabilities Res Rev 14(2):77-86

4. Movahedi F, Kurosu A, Coyle JL, Perera S, Sejdić E (2017) Anatomical directional dissimilarities in tri-axial swallowing accelerometry signals. IEEE Trans Neural Syst Rehabil Eng 25(5):447-458 
5. Clavé $P$, Terré R, de Kraa $M$, Serra $M$ (2004) Approaching oropharyngeal dysphagia. Rev Esp Enferm Dig 96(2):119-131

6. Cook IJ, Kahrilas PJ (1999) AGA technical review on management of oropharyngeal dysphagia. Gastroenterology 116(2):455-478

7. Gordon C, Hewer RL, Wade DT (1987) Dysphagia in acute stroke. Br Med J 295(6595):411-414

8. Humbert IA, Robbins J (2008) Dysphagia in the elderly. Phys Med Rehabilit Clin North Am 19(4):853-866

9. Gorell JM, Johnson CC, Rybicki BA (1994) Parkinson's disease and its comorbid disorders An analysis of Michigan mortality data, 1970 to 1990 . Neurology 44(10):1865-1865

10. Bhattacharyya N (2014) The prevalence of dysphagia among adults in the United States. Otolaryngol-Head Neck Surg 151(5):765-769

11. Murray J (1999) Manual of dysphagia assessment in adults. Cengage Learning

12. Lazarus C, Logemann A (1987) Swallowing disorders in closed head trauma patients. Arch Phys Med Rehabil 68(2):79-84

13. Logemann JA (1998) Evaluation and treatment of swallowing disorders

14. Langmore SE, Kenneth SM, Olsen N (1988) Fiberoptic endoscopic examination of swallowing safety: a new procedure. Dysphagia 2(4):216-219

15. Coyle JL, Davis LA, Easterling C, Graner DE, Langmore S, Leder SB, Lefton-Greif MA, Leslie P, Logemann JA, Mackay L, MartinHarris B, Murray JT, Sonies B, Steele CM (2009) Oropharyngeal dysphagia assessment and treatment efficacy: setting the record straight (response to Campbell-Taylor). J Am Med Dir Assoc 10(1):62-66

16. Rugiu M (2007) Role of videofluoroscopy in evaluation of neurologic dysphagia. Acta Otorhinolaryngol Ital 27(6):306

17. Reddy NP, Katakam A, Gupta V, Unnikrishnan R, Narayanan J, Canilang EP (2000) Measurements of acceleration during videofluorographic evaluation of dysphagic patients. Med Eng Phys 22(6):405-412

18. Martin-Harris B, Jones B (2008) The videofluorographic swallowing study. Phys Med Rehabil Clin North Am 19(4):769-785

19. Logemann JA (1999) Behavioral management for oropharyngeal dysphagia. Folia Phoniatrica et Logopaedica 51(4-5):199-212

20. Steele CM, Alsanei WA, Ayanikalath S, Barbon CE, Chen J, Cichero JA, Coutts K, Dantas RO, Duivestein J, Giosa L, Hanson B, Lam P, Lecko C, Leigh C, Nagy A, Namasivayam AM, Nascimento WV, Odendaal I, Smith CH, Wang H (2015) The influence of food texture and liquid consistency modification on swallowing physiology and function: a systematic review. Dysphagia 30(1):2-26

21. Schünke M, Schulte E, Schumacher U, Ross LM, Lamperti ED (2006) Thieme atlas of anatomy: general anatomy and musculoskeletal system. Thieme Stuttgart, vol 1

22. Coyle JL (2021) Biomechanical analysis. Videofluoroscopy: A multidisciplinary team approach, pp 107-121

23. Kim Y, McCullough GH (2008) Maximum hyoid displacement in normal swallowing. Dysphagia 23(3):274-279

24. Ishida R, Palmer JB, Hiiemae KM (2002) Hyoid motion during swallowing: factors affecting forward and upward displacement. Dysphagia 17(4):262-272

25. Steele CM, Bailey GL, Chau T, Molfenter SM, Oshalla M, Waito AA, Zoratto DC (2011) The relationship between hyoid and laryngeal displacement and swallowing impairment. Clin Otolaryngol 36(1):30-36

26. Feng $X$, Todd $T$, Hu Y, Lintzenich CR, Carr JJ, Browne JD, Kritchevsky SB, Butler SG (2014) Age-related changes of hyoid bone position in healthy older adults with aspiration. Laryngoscope 124(6):E231-E236

27. Kim Y, McCullough GH (2010) Maximal hyoid excursion in poststroke patients. Dysphagia 25(1):20-25

28. Seo HG, Oh B-M, Han TR (2016) Swallowing kinematics and factors associated with laryngeal penetration and aspiration in stroke survivors with dysphagia. Dysphagia 31(2):160-168

29. Molfenter SM, Steele CM (2014) Kinematic and temporal factors associated with penetration-aspiration in swallowing liquids. Dysphagia 29(2):269-276

30. Rosenbek JC, Robbins JA, Roecker EB, Coyle JL, Wood JL (1996) A penetration-aspiration scale. Dysphagia 11(2):93-98

31. Hanley JA, Negassa A, Forrester JE (2003) Statistical analysis of correlated data using generalized estimating equations: an orientation. Am J Epidemiol 157(4):364-375

32. Ziegler A, Vens $M(2010)$ Generalized estimating equations. Methods Inf Med 49(5):421-425

33. Sloane D, Morgan SP (1996) An introduction to categorical data analysis. Ann Rev Sociol 22(1):351-375

34. Steele CM, Cichero JA (2014) Physiological factors related to aspiration risk: a systematic review. Dysphagia 29(3):295-304

35. Wang T-G, Chang Y-C, Chen W-S, Lin P-H, Hsiao T-Y (2010) Reduction in hyoid bone forward movement in irradiated nasopharyngeal carcinoma patients with dysphagia. Arch Phys Med Rehabil 91(6):926-931

36. Butler SG, Stuart A, Leng $X$, Rees C, Williamson J, Kritchevsky SB (2010) Factors influencing aspiration during swallowing in healthy older adults. Laryngoscope 120(11):2147-2152

37. Daggett A, Logemann J, Rademaker A, Pauloski B (2006) Laryngeal penetration during deglutition in normal subjects of various ages. Dysphagia 21(4):270-274

38. Robbins J, Coyle J, Rosenbek J, Roecker E, Wood J (1999) Differentiation of normal and abnormal airway protection during swallowing using the penetration-aspiration scale. Dysphagia 14(4):228-232

39. Rofes L, Arreola V, Mukherjee R, Swanson J, Clavé P (2014) The effects of a xanthan gum-based thickener on the swallowing function of patients with dysphagia. Aliment Pharmacol Ther 39(10):1169-1179

40. Logemann JA, Gensler G, Robbins J, Lindblad AS, Brandt D, Hind JA, Kosek S, Dikeman K, Kazandjian M, Gramigna GD et al (2008) A randomized study of three interventions for aspiration of thin liquids in patients with dementia or Parkinson's disease. J Speech Lang Hear Res 51(1):173-183

41. Newman R, Vilardell N, Clavé P, Speyer R (2016) Effect of bolus viscosity on the safety and efficacy of swallowing and the kinematics of the swallow response in patients with oropharyngeal dysphagia: white paper by the European Society for Swallowing Disorders (ESSD). Dysphagia 31(2):232-249

42. Yucel A, Unlu M, Haktanir A, Acar M, Fidan F (2005) Evaluation of the upper airway cross-sectional area changes in different degrees of severity of obstructive sleep apnea syndrome: cephalometric and dynamic ct study. Am J Neuroradiol 26(10):2624-2629

43. Thapa A, Jayan B, Nehra K, Agarwal S, Patrikar S, Bhattacharya $D$ (2015) Pharyngeal airway analysis in obese and non-obese patients with obstructive sleep apnea syndrome. Med J Armed Forces India 71:S369-S375

Publisher's Note Springer Nature remains neutral with regard to jurisdictional claims in published maps and institutional affiliations. 